\title{
Performance of In-Band Transmission of System Information in Massive MIMO Systems
}

Marcus Karlsson, Emil Björnson and Erik G Larsson

The self-archived postprint version of this journal article is available at Linköping University Institutional Repository (DiVA):

http:/ / urn.kb.se/ resolve?urn=urn:nbn:se:liu:diva-147125

N.B.: When citing this work, cite the original publication.

Karlsson, M., Björnson, E., Larsson, E. G, (2018), Performance of In-Band Transmission of System Information in Massive MIMO Systems, IEEE Transactions on Wireless Communications, 17(3), 1700-1712. https:// doi.org/ 10.1109/TWC.2017.2784809

Original publication available at:

https:// doi.org/ 10.1109/TWC.2017.2784809

Copyright: Institute of Electrical and Electronics Engineers (IEEE)

http:/ / www.ieee.org/ index.html

(C) 2018 IEEE. Personal use of this material is permitted. However, permission to reprint/ republish this material for advertising or promotional purposes or for creating new collective works for resale or redistribution to servers or lists, or to reuse any copyrighted component of this work in other works must be obtained from the IEEE. 


\title{
Performance of In-band Transmission of System Information in Massive MIMO Systems
}

\author{
Marcus Karlsson, Emil Björnson and Erik G. Larsson
}

\begin{abstract}
We consider transmission of system information in massive MIMO. This information needs to be reliably delivered to inactive users in the cell without any channel state information at the base station. Downlink transmission entails the use of downlink pilots and a special type of precoding that aims to reduce the dimension of the downlink channel and the pilot overhead, which would otherwise scale with the number of base station antennas. We consider a scenario in which the base station transmits over a small number of coherence intervals, providing little time/frequency diversity. The system information is transmitted with orthogonal space-time block codes to increase reliability and performance is measured using outage rates. Several different codes are compared, both for spatially correlated and uncorrelated channels and for varying amount of time/frequency diversity. We show that a massive MIMO base station can outperform a single-antenna base station in all considered scenarios.
\end{abstract}

\section{INTRODUCTION}

Massive MIMO (Multiple-Input Multiple-Output) can bring impressive gains in spectral efficiency, quality of service and fairness compared with contemporary wireless communication systems [1]-[3]. Advanced testbeds [3]-[5] are already confirming that the theoretical gains and benefits of massive MIMO can be reaped in practical settings. However, there are still significant problems that need to be solved in order to make it the key technology of the next generation cellular networks. In particular, the base station (BS) needs some way to convey information about cell operation, such as carrier frequencies, bandwidths, and configurations-commonly called system information (SI) - to the terminals in the cell. This transmission of SI is needed for initial access-when an inactive terminal joins the network - and for handover operations. Many papers focus on analyzing the benefits of the technology in the physical layer when the terminals have already received the SI and are regularly transmitting uplink pilots. Conveying SI in massive MIMO has been considered a problem by many in the community and even a show-stopper by some [6].

When the BS has channel state information (CSI), it is able to perform beamforming to achieve a coherent array gain, effectively increasing the signal-to-noise-ratio (SNR) at the receiving terminals. This means that, when CSI is available, more terminals can be reached compared to contemporary single-antenna systems, without increasing the transmit power.

The authors are with the Department of Electrical Engineering (ISY), Linköping University, 58183 Linköping, Sweden (email: \{marcus.karlsson, emil.bjornson, erik.g.larsson\} @liu.se).

This work was supported in part by the Swedish Research Council (VR), and ELLIIT.

A preliminary version of parts of this work was presented at the International Symposium on Wireless Communication Systems (ISWCS), 2015 [17].
However, when the BS does not have CSI, this array gain is lost. Consequently, there is a gap in the received signal power between the signal carrying SI, transmitted without CSI, and the stronger user-dedicated signal, transmitted with CSI. As a result, the area the BS can cover without CSI is smaller than the area covered with CSI.

A space-time block code (STBC) can improve the reliability of transmission without CSI by increasing the effective SNR at the receiver and by providing spatial diversity. Many contemporary systems use STBCs, but massive MIMO offers more freedom in choosing a code because of the larger number of antennas. One specific choice of a STBC is called beam sweeping [7], where the BS sweeps over the cell with the same message using different beams in order to find the terminal. The more antennas the BS has, the narrower beams it can use, resulting in a high received SNR whenever the beam "hits" the terminal. However, beam sweeping is essentially a spatial repetition code-hence, inefficient.

In this paper, we mainly consider scenarios with stringent latency constraints, high reliability requirements, and a channel that offers little or no time/frequency diversity. A representative scenario could be a narrow-band channel in a cellular system handling SI or a sensor network using lowenergy, narrow-band sensors. We consider using an orthogonal STBC (OSTBC) which enables full diversity and simple decoding, both desirable in the above-mentioned scenarios. To enable downlink training, a precoding matrix is used to reduce the pilot overhead. Moreover, only in-band solutions are considered, for which the SI is transmitted in the same frequency band as the payload data.

\section{A. Related Work and Contributions}

Transmission of SI in massive MIMO has been considered in [8]-[11] and is of concern to the industry [12]. Reference [8] presents the need for a precoding matrix to reduce pilot overhead, and focuses on optimizing this precoding matrix, constructed from Zadoff-Chu sequences, to achieve approximate omnidirectional transmission on the average. Here, approximate means that the signal powers in all of the $M$ equally spaced discrete angles are identical. The article also measures system performance in terms of the peak-to-average-power ratio of the transmitted signal, outage probability, and ergodic rate when the user has perfect CSI. In [9], the same authors design the STBC and the precoding matrix jointly, to achieve approximate omnidirectional transmission in each channel use. In [10], [11] omnidirectional transmission, where signal power is constant for any angle, not just discrete ones, is considered. 
The design in [11] allows for small fluctuations in average power over the angles, while [10] considers omnidirectional transmission, averaged over a few channel uses. Any of these methods regarding SI can be used together with the method proposed in [13], where SI is transmitted in the same timefrequency resource as the payload data but is confined to the nullspace of the beamforming matrix used for the payload data.

Note that, although all users in the cell receive the same message from the BS, there is a clear distinction between transmitting SI and multi-casting in massive MIMO. When multi-casting [14], the BS exploits CSI in order to beamform the common information to the terminals. There are also some minor similarities with reducing the dimension of the channel, as done in this paper, and what is known as hybrid beamforming [15], where the BS uses a low dimensional digital precoder and maps the output of this to the antenna array with a high dimensional analogue precoder, consisting of phase shifters. Some prominent differences between hybrid beamforming and dimension reduction are: hybrid beamforming is limited by the number of RF chains, but in this paper, each antenna has its own RF chain; many of the algorithms used in hybrid beamforming aim to maximize the spectral efficiency, ignoring the users with poor channel conditions; and hybrid beamforming needs CSI which is not available to the BS in the scenario considered here. Additionally, there is no guarantee that the dimension reduction with a given STBC can be realized using hybrid beamforming.

The specific contributions of the paper are the following:

- We derive a lower bound on the SNR obtained at the terminal for downlink communication in a massive MIMO system using downlink pilots and an arbitrary OSTBC without any prior CSI available to the terminal or the BS. This bound is found to be close to a bound that follows as a special case of the results in [13], where no structure of the transmitted signal is assumed.

- We analyze the need for spatial diversity for transmission of SI in a massive MIMO system by comparing the performance of several OSTBCs in correlated and uncorrelated channels. For the considered scenario, using codes providing a higher diversity order than around 10 is not beneficial. For larger codes the increase in spatial diversity is not enough to counteract the pre-log penalty associated with the pilot overhead.

- We study how the availability of time-frequency resources for SI affects the choice of OSTBCs. Here we consider two cases: First, the amount of information the BS wants to convey to the terminal is fixed and the BS minimizes the amount of time-frequency resources used. Second, the amount of time-frequency resources available for SI is fixed and the BS aims to convey as much information as possible to the terminal.

- We derive a corresponding lower bound on the SNR at the terminal, for the case of a multi-cell system with different pilot reuse, and compare performance to that of the single-cell system.

In earlier conference papers we have presented some initial results. In [16], we highlighted the need for downlink pilots for transmission without CSI at the BS and introduced the idea of spatially repeating a small code over the antennas. Reference [17] treated a scenario similar to the one in the current paper, but the analysis here is includes correlated channels, larger and rectangular OSTBCs, least-squares estimation, pilot-energy optimization, and multiple cells.

Notation: Boldfaced lowercase letters, x, denote column vectors, boldface uppercase letters, $\mathbf{X}$, denote matrices and lower case letters, $x$, denote scalars. $\mathbf{I}_{M}$ is the identity matrix of dimension $M \times M$ and $\mathbf{0}_{a \times b}$ is the zero matrix of dimensions $a \times b . \mathbf{X}^{*}, \mathbf{X}^{\mathrm{T}}$ and $\mathbf{X}^{\mathrm{H}}$ denote conjugate, transpose and Hermitian transpose, respectively. The 2 -norm of a vector $\mathbf{x}$ is denoted by $\|\mathbf{x}\| . \bar{x} \triangleq \Re\{x\}$ and $\tilde{x} \triangleq \Im\{x\}$ denote the real and imaginary parts, respectively, and the imaginary unit is denoted by i. $\mathcal{C N}(\mathbf{x}, \mathbf{X})$ represents the circularly symmetric, complex Gaussian distribution with mean $\mathbf{x}$ and covariance matrix $\mathbf{X}$ and $\chi^{2}(m)$ is a Chi-squared distribution with $m$ degrees of freedom. The notation $f(x)=\mathcal{O}(g(x))$ means that there exist positive constants $c$ and $x_{0}$ such that

$$
|f(x)| \leq c|g(x)|, \forall x \geq x_{0} .
$$

\section{BACKGROUND}

\section{A. Orthogonal Space-Time Block Codes}

This subsection introduces OSTBCs and their associated terminology, starting with the more general linear STBCs. The information in this section can be found in, for example [18], but some key equations are stated here in order to make the paper self-contained as well as to establish notation and terminology.

A linear STBC is a code for which each code matrix (codeword) $\mathrm{X}$ carries $n_{\mathrm{S}}$ information bearing symbols over $\tau$ channel uses, using $n_{\mathrm{T}}$ antennas. That is, each code matrix $\mathbf{X}$ is a $\tau \times n_{\mathrm{T}}$ (complex-valued) matrix of the form

$$
\mathbf{X}=\sum_{n=1}^{n_{\mathrm{s}}} \bar{s}_{n} \mathbf{A}_{n}+\mathrm{i} \tilde{s}_{n} \mathbf{B}_{n},
$$

where $\bar{s}_{n}\left(\tilde{s}_{n}\right)$ is the real (imaginary) part of the symbol to be transmitted, $s_{n}=\bar{s}_{n}+\mathrm{i} \tilde{s}_{n} . \mathbf{A}_{n}$ and $\mathbf{B}_{n}$ are fixed $\tau \times n_{\mathrm{T}}$, generally complex-valued, matrices which define the specific code. Since $n_{\mathrm{S}}$ symbols are conveyed over $\tau$ channel uses, the code rate is $n_{\mathrm{S}} / \tau$. We also refer to $\tau$ as the decoding delay, or simply delay, since the receiver has to wait $\tau$ channel uses before decoding the codeword $\mathbf{X}$. We will further refer to $n_{\mathrm{T}}$ as the size of the code. Specifically, a "larger code" means a code with larger $n_{\mathrm{T}}$.

An OsTBC is a linear STBC for which all code matrices satisfy

$$
\mathbf{X}^{\mathrm{H}} \mathbf{X}=\sum_{n=1}^{n_{\mathrm{s}}}\left|s_{n}\right|^{2} \mathbf{I}_{n_{\mathrm{T}}} .
$$

This implies that $\tau \geq n_{\mathrm{T}}$. This orthogonality also means that the symbols decouple in coherent detection [18, Section 7.4], [19]. 
TABLE I

THE PARAMETERS OF THE OSTBCS CONSIDERED IN THE PAPER.

\begin{tabular}{|c|c|c|c|c|}
\hline Code ID & $n_{\mathrm{T}}$ & $\tau_{\mathrm{D}}$ & $n_{\mathrm{S}}$ & Code Rate $\left(\frac{n_{\mathrm{S}}}{\tau_{\mathrm{D}}}\right)$ \\
\hline 1 & 1 & 1 & 1 & 1 \\
\hline 2 & 2 & 2 & 2 & 1 \\
\hline 4 & 4 & 4 & 3 & $3 / 4$ \\
\hline 8 & 8 & 16 & 8 & $1 / 2$ \\
\hline 12 & 12 & 128 & 64 & $1 / 2$ \\
\hline
\end{tabular}

All OSTBCs satisfy the following identities [18, Theorem 7.1]:

$$
\begin{aligned}
& \mathbf{A}_{n}^{\mathrm{H}} \mathbf{A}_{n}=\mathbf{I}_{n_{\mathrm{T}}}, \mathbf{B}_{n}^{\mathrm{H}} \mathbf{B}_{n}=\mathbf{I}_{n_{\mathrm{T}}}, \\
& \mathbf{A}_{n}^{\mathrm{H}} \mathbf{A}_{k}=-\mathbf{A}_{k}^{\mathrm{H}} \mathbf{A}_{n}, \mathbf{B}_{n}^{\mathrm{H}} \mathbf{B}_{k}=-\mathbf{B}_{k}^{\mathrm{H}} \mathbf{B}_{n}, \quad \forall n, k, n \neq k, \\
& \mathbf{A}_{n}^{\mathrm{H}} \mathbf{B}_{k}=\mathbf{B}_{k}^{\mathrm{H}} \mathbf{A}_{n}, \quad \forall n, k .
\end{aligned}
$$

From these identities one can deduce that for any complexvalued vector $\mathbf{v}$

$$
\Re\left\{\mathbf{v}^{\mathrm{H}} \mathbf{A}_{n}^{\mathrm{H}} \mathbf{A}_{k} \mathbf{v}\right\}= \begin{cases}0, & n \neq k \\ \|\mathbf{v}\|^{2}, & n=k\end{cases}
$$

and

$$
\Re\left\{-\mathbf{i} \mathbf{v}^{\mathrm{H}} \mathbf{A}_{n}^{\mathrm{H}} \mathbf{B}_{k} \mathbf{v}\right\}=\Im\left\{\mathbf{v}^{\mathrm{H}} \mathbf{A}_{n}^{\mathrm{H}} \mathbf{B}_{k} \mathbf{v}\right\}=0, \forall n, k,
$$

which will prove useful later.

As a special case of (1), consider letting $s_{n}=s$ for $n=$ $1, \ldots, n_{\mathrm{s}}$, then

$$
\mathbf{X}=\mathbf{C} s,
$$

for some complex matrix $\mathbf{C}$. This is one way of describing beam sweeping, where the rows of $\mathbf{C}$ are designed to provide spatial coverage. We see here that beam sweeping is a special case of a linear STBC with code rate $1 / \tau$.

In this paper, we consider four different OstBCs. As a reference, we also consider a BS with a single antenna. The considered OSTBCs are listed and summarized in Table I. When referring to the codes, we will use the code identity (ID), defined in Table I. Code 2 is the Alamouti code [20] and code 4 can be found in [18, Example 7.4]. Codes 8 and 12 were created following the algorithm outlined in [19].

The two larger codes in Table I are suboptimal, both in terms of rate [21] and delay [22]. This guarantees that an optimal code (in terms of rate, delay, or both) will perform at least as well. The main point, however, is that a massive MIMO BS can outperform a single-antenna BS and to show this, the codes in Table I are more than enough.

\section{B. The Finite Coherence Interval}

The coherence interval is a time-frequency block whose time-duration is equal to the coherence time and whose bandwidth is equal to the coherence bandwidth. The size of the coherence interval in samples, denoted $\tau_{\mathrm{C}}$, can vary vastly between applications, from a few hundred symbols, to practically infinite [1, Chapter 2]. For an inactive user, the BS does not know the length of the coherence interval, and hence has to use a conservative estimate in order to reduce the risk of overestimating the stability of the channel. In practice, the system is limited by the channel offering the smallest coherence interval.

The finite coherence interval is the reason why massive MIMO requires time-division duplex (TDD) operation in order to be scalable in the number of BS antennas, unless additional assumptions on propagation are made [6]. TDD enables channel reciprocity within a coherence interval, which allows the BS to learn the uplink and downlink channels from uplink pilots. If downlink pilots were used, a BS with $M$ antennas would have to spend at least $M$ channel uses on downlink training in every coherence interval, plus additional feedback.

\section{SYSTEM MODEL}

The paper will focus on the single-cell case, where no interference from other cells is present, as most of the interesting phenomena arise there. However, we will provide a brief discussion of what changes in a multi-cell scenario in Section III-D and compare some of the results for the singlecell scenario to that for the multi-cell scenario.

Consider a single-cell system in which the BS is equipped with $M$ antennas and wishes to convey si to an arbitrary single-antenna user within the cell. Neither the BS nor the terminal has any a priori CSI. The received signal at the terminal is

$$
y \triangleq \sqrt{\rho} \mathbf{x}^{\mathrm{T}} \mathbf{g}+w,
$$

where $\mathbf{x} \in \mathbb{C}^{M \times 1}, \mathbf{g} \in \mathbb{C}^{M \times 1}$, and $w$ are the transmitted signal, the channel, and noise, respectively. The transmitted signal $\mathbf{x}$ satisfies $\mathbb{E}\left[\mathbf{x}^{\mathrm{H}} \mathbf{x}\right]=1, \rho$ is the normalized transmit power and $w \sim \mathcal{C N}(0,1)$ is independent, normalized noise. The channel $\mathrm{g}$ is assumed to be distributed as $\mathcal{C N}\left(\mathbf{0}, \mathbf{C}_{\mathrm{g}}\right)$, where $\mathbf{C}_{\mathbf{g}} \triangleq \mathbb{E}\left[\mathrm{gg}^{\mathrm{H}}\right] \in \mathbb{C}^{M \times M}$ is the channel covariance matrix. Over $\tau$ channel uses the BS transmits the $\tau \times M$ matrix

$$
\mathbf{X} \triangleq\left[\begin{array}{c}
\mathbf{x}_{1}^{\mathrm{T}} \\
\mathbf{x}_{2}^{\mathrm{T}} \\
\vdots \\
\mathbf{x}_{\tau}^{\mathrm{T}}
\end{array}\right]
$$

whereby the user receives the $\tau \times 1$ vector

$$
\mathbf{y} \triangleq \sqrt{\rho} \mathbf{X g}+\mathbf{w}
$$

where

$$
\mathbf{w} \triangleq\left[w_{1}, \ldots, w_{\tau}\right]^{\mathrm{T}}
$$

has independent $\mathcal{C N}(0,1)$ elements.

When the user detects the transmitted symbols, it is beneficial to have CSI. To give the terminal CSI, the BS first transmits the pilot matrix $\mathbf{X}_{\mathrm{P}} \in \mathbb{C}^{\tau_{\mathrm{P}} \times M}$, known a priori to both parties. Orthogonal pilots $\left(\mathbf{X}_{\mathrm{P}}^{\mathrm{H}} \mathbf{X}_{\mathrm{P}} \propto \mathbf{I}_{M}\right)$ are usually preferred as they are optimal in a mean-squared-error sense [18, Section 9.4] in independent, identically distributed (i.i.d.) Rayleigh fading. Additionally, orthogonal pilots ensure that the channel coefficients decouple during estimation in i.i.d. Rayleigh fading. However, transmitting orthogonal pilots would require $\tau_{\mathrm{P}} \geq M$, which means spending many channel uses on pilots. If $\tau_{\mathrm{P}}$ is of the same order as the coherence interval $\tau_{\mathrm{C}}$, few channel uses will be left for data, and if $\tau_{\mathrm{P}}>\tau_{\mathrm{C}}$, the orthogonal pilot block would be too big to fit in one coherence interval. 
Transmitting SI is seemingly the only time, apart from a computational complexity perspective, when a massive MIMO system does not benefit from having more antennas. If the BS only had a few antennas, there would be no problem sending orthogonal downlink pilots. To resolve this problem, there are a few alternatives: i) Restrict the number of antennas at the BS for the sole purpose of being able to transmit orthogonal downlink pilots when conveying SI. This is not an appealing solution since it eliminates the benefits of massive MIMO. ii) Turn off antennas and transmit SI on only a subset of the array. This is problematic because either the transmission without CSI will have to be done with a fraction of the total output power used in coherent transmission, or the hardware has to be able to work with large variations in output power, which would make the hardware more expensive. iii) Make use of the excess of degrees of freedom and spatial diversity, provided by the abundance of antennas at the BS. iv) Use a single, more powerful antenna operating at another frequency, dedicated to provide SI. As this paper only considers in-band solutions, option iv is out of scope.

We consider the third alternative, and aim to find a middle ground between full repetition over the antennas (beam sweeping), associated with a lower rate, and no repetition, associated with a large pilot overhead.

\section{A. The Dimension Reducing Matrix}

As mentioned earlier in Section III, having a BS with a moderate or even small number of antennas might be beneficial, considering the same total output power. To emulate a BS with few antennas, consider constructing the transmitted signal $\mathbf{X}$ with two separate parts:

$$
\mathbf{X}=\mathbf{X} \Phi
$$

where $\boldsymbol{\Phi} \in \mathbb{C}^{n_{\mathrm{T}} \times M}, n_{\mathrm{T}}<M$ is a (deterministic) precoding matrix called the dimension-reducing matrix (DRM), with the purpose of spreading the OsTBC $\mathbf{X} \in \mathbb{C}^{\tau \times n_{\mathrm{T}}}$ over the antennas. With (5), the received signal (4) can be written as

$$
\mathbf{y}=\sqrt{\rho} \mathbf{X} \mathbf{\Phi g}+\mathbf{w}=\sqrt{\rho} \mathbf{X h}+\mathbf{w},
$$

where we have defined the effective channel $\mathbf{h} \triangleq \mathbf{\Phi g} \in \mathbb{C}^{n_{\mathrm{T}}}$. The DRM effectively shrinks the channel dimension from $M$ to $n_{\mathrm{T}}$. The matrix $\mathbf{X}$ can be thought of as the output of $n_{\mathrm{T}}$ antenna ports, and $\boldsymbol{\Phi}$ represents the mapping from the antenna ports to the physical antennas.

After choosing a DRM, the BS can transmit SI to the users in the cell over the effective channel $\mathbf{h}$. The transmission is divided into a pilot phase, in which the BS transmits a predetermined set of pilots in the downlink, and a data phase, in which information-bearing symbols are transmitted. Note that the DRM has to remain constant for the entire coherence interval, i.e., over both the pilot and the data phase.

\section{B. Pilot Phase}

As long as the DRM is fixed within a coherence interval, the effective channel $\mathbf{h}$ is static, which means it can be estimated.
To estimate the channel, a semi-unitary pilot matrix $\mathbf{X}_{\mathrm{P}} \in$ $\mathbb{C}^{\tau_{\mathrm{P}} \times n_{\mathrm{T}}}, \tau_{\mathrm{P}} \geq n_{\mathrm{T}}$, satisfying

$$
\mathbf{X}_{\mathrm{P}}^{\mathrm{H}} \mathbf{X}_{\mathrm{P}}=\frac{\tau_{\mathrm{P}}}{n_{\mathrm{T}}} \mathbf{I}_{n_{\mathrm{T}}}
$$

is transmitted with normalized transmit power $\rho_{\mathrm{P}}$ by the BS. The received signal at the terminal is

$$
\mathbf{y}_{\mathrm{P}} \triangleq \sqrt{\rho_{\mathrm{P}}} \mathbf{X}_{\mathrm{P}} \mathbf{h}+\mathbf{w}
$$

Since the terminal lacks CSI, the least-squares estimate of the channel is used:

$$
\hat{\mathbf{h}} \triangleq\left(\sqrt{\rho_{\mathrm{P}}} \mathbf{X}_{\mathrm{P}}^{\mathrm{H}} \mathbf{X}_{\mathrm{P}}\right)^{-1} \mathbf{X}_{\mathrm{P}}^{\mathrm{H}} \mathbf{y}_{\mathrm{P}}=\mathbf{h}+\mathbf{e}
$$

where

$$
\mathbf{e} \triangleq\left(\sqrt{\rho_{\mathrm{P}}} \mathbf{X}_{\mathrm{P}}^{\mathrm{H}} \mathbf{X}_{\mathrm{P}}\right)^{-1} \mathbf{X}_{\mathrm{P}}^{\mathrm{H}} \mathbf{w}=\hat{\mathbf{h}}-\mathbf{h}
$$

is the channel estimation error. The channel and the estimation error have covariance matrices

$$
\mathbf{C}_{\mathbf{h}} \triangleq \mathbb{E}\left[\mathbf{h h}^{\mathrm{H}}\right]=\mathbb{E}\left[\boldsymbol{\Phi} \mathbf{g g}^{\mathrm{H}} \boldsymbol{\Phi}^{\mathrm{H}}\right]=\boldsymbol{\Phi} \mathbf{C}_{\mathbf{g}} \boldsymbol{\Phi}^{\mathrm{H}}
$$

and

$$
\mathbf{C}_{\mathbf{e}} \triangleq \mathbb{E}\left[\mathbf{e e}^{\mathrm{H}}\right]=\frac{n_{\mathrm{T}}}{\rho_{\mathrm{P}} \tau_{\mathrm{P}}} \mathbf{I}_{n_{\mathrm{T}}}
$$

respectively.

The channel estimate, $\hat{\mathbf{h}}$, and the channel estimation error, e, are circularly symmetric complex variables. They are moreover jointly Gaussian and correlated, which implies that we can write [23, Theorem 10.2]

$$
\mathbf{e} \mid \hat{\mathbf{h}} \sim \mathcal{C N}(\mathbf{U} \hat{\mathbf{h}}, \mathbf{R})
$$

where

$$
\mathbf{U} \triangleq \mathbf{C}_{\mathbf{e}}\left(\mathbf{C}_{\mathbf{e}}+\mathbf{C}_{\mathbf{h}}\right)^{-1}, \text { and } \mathbf{R} \triangleq\left(\mathbf{C}_{\mathbf{e}}^{-1}+\mathbf{C}_{\mathbf{h}}^{-1}\right)^{-1} .
$$

In particular, this means that

$$
\mathbb{E}\left[\mathbf{e} \mathbf{e}^{\mathrm{H}} \mid \hat{\mathbf{h}}\right]=\mathbf{R}+\mathbf{U} \hat{\mathbf{h}} \hat{\mathbf{h}}^{\mathrm{H}} \mathbf{U}^{\mathrm{H}} \text { and } \mathbb{E}\left[\mathbf{e} \mathbf{e}^{\mathrm{T}} \mid \hat{\mathbf{h}}\right]=\mathbf{U} \hat{\mathbf{h}} \hat{\mathbf{h}}^{\mathrm{T}} \mathbf{U}^{\mathrm{T}} \text {. }
$$

\section{Data Phase}

In the data phase, the BS transmits a number of OSTBC matrices $\mathbf{X}_{\mathrm{D}} \in \mathbb{C}^{\tau_{\mathrm{D}} \times n_{\mathrm{T}}}$, conveying $n_{\mathrm{S}}$ mutually independent information-bearing symbols over $\tau_{\mathrm{D}}$ channel uses. With $\rho_{\mathrm{D}}$ denoting the normalized transmit power, the received signal at the terminal is

$$
\mathbf{y}=\sqrt{\rho_{\mathrm{D}}} \mathbf{X}_{\mathrm{D}} \mathbf{h}+\mathbf{w}
$$

The codeword $\mathbf{X}_{\mathrm{D}}$ satisfies

$$
\mathbb{E}\left[\operatorname{tr}\left(\mathbf{X}_{\mathrm{D}}^{\mathrm{H}} \mathbf{X}_{\mathrm{D}}\right)\right]=\tau_{\mathrm{D}},
$$

and the symbol energy is

$$
E_{s} \triangleq \mathbb{E}\left[\left|s_{n}\right|^{2}\right]=\frac{\tau_{\mathrm{D}}}{n_{\mathrm{S}} n_{\mathrm{T}}} .
$$

In order to detect the complex symbol $s_{n}$, the user treats the estimated channel $\hat{\mathbf{h}}$ as the true channel and detects the real and imaginary part of $s_{n}$ separately. To detect the real part of the transmitted symbol, $\bar{s}_{n}$, the terminal multiplies the 
received vector with $\hat{\mathbf{h}}^{\mathrm{H}} \mathbf{A}_{n}^{\mathrm{H}}$ from the left and takes the real part [18]:

$$
\begin{aligned}
\hat{\bar{s}}_{n} & =\Re\left\{\hat{\mathbf{h}}^{\mathrm{H}} \mathbf{A}_{n}^{\mathrm{H}} \mathbf{y}\right\} \\
& =\Re\left\{\sqrt{\rho_{\mathrm{D}}} \hat{\mathbf{h}}^{\mathrm{H}} \mathbf{A}_{n}^{\mathrm{H}} \mathbf{X}_{\mathrm{D}} \hat{\mathbf{h}}-\sqrt{\rho_{\mathrm{D}}} \hat{\mathbf{h}}^{\mathrm{H}} \mathbf{A}_{n}^{\mathrm{H}} \mathbf{X}_{\mathrm{D}} \mathbf{e}+\hat{\mathbf{h}}^{\mathrm{H}} \mathbf{A}_{n}^{\mathrm{H}} \mathbf{w}\right\} .
\end{aligned}
$$

From (2) and (3),

$$
\Re\left\{\sqrt{\rho_{\mathrm{D}}} \hat{\mathbf{h}}^{\mathrm{H}} \mathbf{A}_{n}^{\mathrm{H}} \mathbf{X}_{\mathrm{D}} \hat{\mathbf{h}}\right\}=\sqrt{\rho_{\mathrm{D}}}|| \hat{\mathbf{h}} \|^{2} \bar{s}_{n} .
$$

The last two terms in (10) are denoted by

$$
\bar{\eta}_{n, 1} \triangleq-\Re\left\{\sqrt{\rho_{\mathrm{D}}} \hat{\mathbf{h}}^{\mathrm{H}} \mathbf{A}_{n}^{\mathrm{H}} \mathbf{X}_{\mathrm{D}} \mathbf{e}\right\}
$$

and

$$
\bar{\eta}_{n, 2} \triangleq \Re\left\{\hat{\mathbf{h}}^{\mathrm{H}} \mathbf{A}_{n}^{\mathrm{H}} \mathbf{w}\right\} .
$$

We can now write the received, processed, real symbol as

$$
\hat{\bar{s}}_{n}=\left.\sqrt{\rho_{\mathrm{D}}}|| \hat{\mathbf{h}}\right|^{2} \bar{s}_{n}+\bar{\eta}_{n, 1}+\bar{\eta}_{n, 2} .
$$

To decode the imaginary part of $s_{n}$, we use $-i \hat{\mathbf{h}}^{\mathrm{H}} \mathbf{B}_{n}^{\mathrm{H}}$ instead of $\hat{\mathbf{h}}^{\mathrm{H}} \mathbf{A}_{n}^{\mathrm{H}}$ and the following calculations are otherwise identical to what we have above. This calculation gives the error terms

$$
\tilde{\eta}_{n, 1} \triangleq-\Re\left\{-\mathrm{i} \sqrt{\rho_{\mathrm{D}}} \hat{\mathbf{h}}^{\mathrm{H}} \mathbf{B}_{n}^{\mathrm{H}} \mathbf{X}_{\mathrm{D}} \mathbf{e}\right\}=-\Im\left\{\sqrt{\rho_{\mathrm{D}}} \hat{\mathbf{h}}^{\mathrm{H}} \mathbf{B}_{n}^{\mathrm{H}} \mathbf{X}_{\mathrm{D}} \mathbf{e}\right\}
$$

and

$$
\tilde{\eta}_{n, 2} \triangleq \Re\left\{-i \hat{\mathbf{h}}^{\mathrm{H}} \mathbf{B}_{n}^{\mathrm{H}} \mathbf{w}\right\}=\Im\left\{\hat{\mathbf{h}}^{\mathrm{H}} \mathbf{B}_{n}^{\mathrm{H}} \mathbf{w}\right\},
$$

completely analogous to $\bar{\eta}_{n, 1}$ and $\bar{\eta}_{n, 2}$ for the detection of the real part. Finally, we can write the received, processed complex symbol as

$$
\begin{aligned}
\hat{s}_{n} & \triangleq \sqrt{\rho_{\mathrm{D}}}|| \hat{\mathbf{h}} \|^{2}\left(\bar{s}_{n}+\mathrm{i} \tilde{s}_{n}\right)+\bar{\eta}_{n, 1}+\mathrm{i} \tilde{\eta}_{n, 1}+\bar{\eta}_{n, 2}+\mathrm{i} \tilde{\eta}_{n, 2} \\
& =\sqrt{\rho_{\mathrm{D}}}|| \hat{\mathbf{h}} \|^{2} s_{n}+\eta_{n, 1}+\eta_{n, 2},
\end{aligned}
$$

where $\eta_{n, 1} \triangleq \bar{\eta}_{n, 1}+\mathrm{i} \tilde{\eta}_{n, 1}$ and $\eta_{n, 2} \triangleq \bar{\eta}_{n, 2}+\mathrm{i} \tilde{\eta}_{n, 2}$.

Conditioned on the channel estimate, $\hat{\mathbf{h}},(11)$ is a deterministic channel plus noise. The first error term, $\eta_{n, 1}$, stemming from the imperfect channel estimate, is correlated with the symbol of interest $s_{n}$. We can thus write

$$
\eta_{n, 1}=c_{n} s_{n}+u_{n},
$$

where $c_{n} \triangleq \mathbb{E}\left[s_{n}^{*} \eta_{n, 1} \mid \hat{\mathbf{h}}\right] / E_{s}$ and $u_{n}$ is uncorrelated to $s_{n}$. With this, (11) becomes

$$
\hat{s}_{n}=\left(\sqrt{\rho_{\mathrm{D}}}\|\hat{\mathbf{h}}\|^{2}+c_{n}\right) s_{n}+u_{n}+\eta_{n, 2} .
$$

The signal in (12) is now uncorrelated to the noise, conditioned on $\hat{\mathbf{h}}$, and the received SNR is given by [24]

$$
\frac{\left|\mathbb{E}\left[s_{n}^{*} \hat{s}_{n} \mid \hat{\mathbf{h}}\right]\right|^{2}}{E_{s} \mathbb{E}\left[\left|\hat{s}_{n}\right|^{2} \mid \hat{\mathbf{h}}\right]-\left|\mathbb{E}\left[s_{n}^{*} \hat{s}_{n} \mid \hat{\mathbf{h}}\right]\right|^{2}} .
$$

With

$$
U_{n} \triangleq \mathbb{E}\left[\left|u_{n}\right|^{2} \mid \hat{\mathbf{h}}\right]=\mathbb{E}\left[\left|\eta_{n, 1}\right|^{2} \mid \hat{\mathbf{h}}\right]-E_{s} \mathbb{E}\left[\left|c_{n}\right|^{2} \mid \hat{\mathbf{h}}\right]
$$

and

$$
\mathbb{E}\left[\left|\eta_{n, 2}\right|^{2} \mid \hat{\mathbf{h}}\right]=\|\hat{\mathbf{h}}\|^{2},
$$

(13) can be expressed as

$$
\mathrm{SNR}_{n} \triangleq \frac{E_{s}\left|\sqrt{\rho_{\mathrm{D}}}\|\hat{\mathbf{h}}\|^{2}+c_{n}\right|^{2}}{U_{n}+\|\left.\hat{\mathbf{h}}\right|^{2}} .
$$

Note that the SNR in (14) can vary between symbols for the same channel realization. This variation in SNR is small: in the order of 0.1 percent for all analyzed cases. We define the achievable SNR when using an OSTBC as

$$
\mathrm{SNR}^{\text {OSTBC }} \triangleq \min _{n \in\left\{1, \ldots, n_{\mathrm{s}}\right\}} \mathrm{SNR}_{n} .
$$

In the special case when the physical channel has i.i.d. elements,

$$
\mathbf{C}_{\mathrm{g}}=\beta \mathbf{I},
$$

where $\beta$ represents the large-scale fading, we have

$$
c_{n}=-\sqrt{\rho_{\mathrm{D}}}|| \hat{\mathbf{h}}||^{2} \frac{n_{\mathrm{T}}}{\beta \tau_{\mathrm{P}} \rho_{\mathrm{P}}+n_{\mathrm{T}}} .
$$

If, in addition, the code is a square OSTBC $\left(n_{\mathrm{T}}=\tau_{\mathrm{D}}\right)$ from Table I,

$$
U_{n}=\frac{\rho_{\mathrm{D}} \tau_{\mathrm{D}} \beta}{\beta \tau_{\mathrm{P}} \rho_{\mathrm{P}}+n_{\mathrm{T}}}\|\hat{\mathbf{h}}\|^{2}
$$

and the symbol SNR in (14) can be simplified to

$$
\mathrm{SNR}^{\text {square }} \triangleq \frac{E_{s} \rho_{\mathrm{D}}\|\hat{\mathbf{h}}\|^{2}}{\frac{\rho_{\mathrm{D}} \tau_{\mathrm{D}} \beta}{n_{\mathrm{T}}+\beta \rho_{\mathrm{P}} \tau_{\mathrm{P}}}+1}\left(\frac{\beta \tau_{\mathrm{P}} \rho_{\mathrm{P}}}{\beta \tau_{\mathrm{P}} \rho_{\mathrm{P}}+n_{\mathrm{T}}}\right)^{2} .
$$

We will later numerically compare the outage rate achieved when using (15) to the rate achieved when using the SNR derived in [13, Eq. (49)], where no structure of the transmitted signal was assumed. The SNR from [13] is given by ${ }^{1}$

$$
\mathrm{SNR}^{\text {general }} \triangleq \frac{\frac{\rho_{\mathrm{D}}}{n_{\mathrm{T}}}\left\|\hat{\mathbf{h}}_{\mathrm{MMSE}}\right\|^{2}}{\frac{n_{\mathrm{T}} \rho_{\mathrm{D}} \beta}{n_{\mathrm{T}}+\tau_{\mathrm{P}} \rho_{\mathrm{P}} \beta}+1},
$$

where $\hat{\mathbf{h}}_{\mathrm{MMSE}}$ is the channel estimate if a minimum-meansquare-error estimator is used by the terminal. The SNR in (17) can be seen as an upper bound on the SNR in (15), as the former does not assume any structure of the transmitted signal.

When a square OSTBC with full rate $\left(n_{\mathrm{S}}=n_{\mathrm{T}}=\tau_{\mathrm{D}}\right)$ is used, (16) and (17) are distributed identically as

$$
\mathrm{SNR}^{\text {square }} \sim \mathrm{SNR}^{\text {general }} \sim \frac{\rho_{\mathrm{P}} \tau_{\mathrm{P}} \rho_{\mathrm{D}} \tau_{\mathrm{D}} \beta^{2}}{2 n_{\mathrm{S}} n_{\mathrm{T}}\left(\rho_{\mathrm{D}} \tau_{\mathrm{D}} \beta+\rho_{\mathrm{P}} \tau_{\mathrm{P}} \beta+n_{\mathrm{T}}\right)} \chi^{2}\left(2 n_{\mathrm{T}}\right) .
$$

This can be shown by observing that

$$
\|\hat{\mathbf{h}}\|^{2} \sim \frac{n_{\mathrm{T}}+\rho_{\mathrm{P}} \tau_{\mathrm{P}} \beta}{2 \rho_{\mathrm{P}} \tau_{\mathrm{P}}} \chi^{2}\left(2 n_{\mathrm{T}}\right)
$$

and

$$
\left\|\hat{\mathbf{h}}_{\mathrm{MMSE}}\right\|^{2} \sim \frac{\rho_{\mathrm{P}} \tau_{\mathrm{P}} \beta^{2}}{2\left(\rho_{\mathrm{P}} \tau_{\mathrm{P}} \beta+n_{\mathrm{T}}\right)} \chi^{2}\left(2 n_{\mathrm{T}}\right) .
$$

${ }^{1}$ In [13], the data power and the pilot power are assumed to be equal, which is not the case here. In addition, we do not consider simultaneous payload transmission, so $\rho_{b}^{\prime}$ is zero. The SNR expression has been modified accordingly. 


\section{The Multi-Cell Scenario}

Deriving the lower bound on the SNR for the multi-cell case follows a similar route as in the single-cell case, only with more terms. We let $K$ denote the number of interfering cells and $\mathcal{K}$ denote the set of contaminating cells that use the same pilots as the home cell. The pilot sequences used by cells not in $\mathcal{K}$ are orthogonal to the pilot sequence used in the home cell. For a pilot reuse of $p$, at least $p n_{\mathrm{T}}$ channel uses will be occupied by pilots.

1) Pilot Phase: Following the same steps as in Section III-B, the multi-cell equivalent to the channel estimate $\hat{\mathbf{h}}$ can be written as

$$
\hat{\mathbf{h}}_{\mathrm{MC}}=\mathbf{h}+\mathbf{e}+\mathbf{h}_{\Sigma},
$$

where

$$
\mathbf{h}_{\Sigma} \triangleq \sum_{k \in \mathcal{K}} \mathbf{h}_{k}
$$

and $\mathbf{h}_{k}$ is the channel from the BS in cell $k$ to the terminal in the home cell. Just as in the single-cell case, the estimation error, now $\mathbf{e}+\mathbf{h}_{\Sigma}$, is correlated to the channel estimate.

To calculate (13) in the multi-cell case, the conditional moments of $\mathbf{h}_{k} \mid \hat{\mathbf{h}}_{\mathrm{MC}}$ for $k=1, \ldots, K, \mathbf{h}_{\Sigma} \mid \hat{\mathbf{h}}_{\mathrm{MC}}$, and $\mathbf{e} \mid \hat{\mathbf{h}}_{\mathrm{MC}}$ are needed, as well as the conditional moments of $\mathbf{e} \mid \hat{\mathbf{h}}_{\mathrm{MC}}, \mathbf{h}_{\Sigma}$. These can be found using [23, Theorem 10.2].

2) Data Phase: When detecting the information-bearing symbol, two additional noise terms show up, compared to the single-cell case:

$$
\eta_{n, 3} \triangleq-\sqrt{\rho_{\mathrm{D}}} \Re\left\{\hat{\mathbf{h}}_{\mathrm{MC}}^{\mathrm{H}} \mathbf{A}_{n}^{\mathrm{H}} \mathbf{X}_{\mathrm{D}} \mathbf{h}_{\Sigma}\right\}-\mathrm{i} \sqrt{\rho_{\mathrm{D}}} \Im\left\{\hat{\mathbf{h}}_{\mathrm{MC}}^{\mathrm{H}} \mathbf{B}_{n}^{\mathrm{H}} \mathbf{X}_{\mathrm{D}} \mathbf{h}_{\Sigma}\right\}
$$

and

$$
\eta_{n, 4} \triangleq \sum_{k=1}^{K} \Re\left\{\hat{\mathbf{h}}_{\mathrm{MC}}^{\mathrm{H}} \mathbf{A}_{n}^{\mathrm{H}} \mathbf{X}_{k} \mathbf{h}_{k}\right\}+\mathrm{i} \Im\left\{\hat{\mathbf{h}}_{\mathrm{MC}}^{\mathrm{H}} \mathbf{B}_{n}^{\mathrm{H}} \mathbf{X}_{k} \mathbf{h}_{k}\right\},
$$

where $\mathbf{X}_{k}$ is the signal transmitted from cell $k$ in the data phase. All cells are assumed to transmit data in the same timefrequency resource. This gives an expression for the received, processed signal in a multi-cell scenario

$$
\hat{s}_{n}=\sqrt{\rho_{\mathrm{D}}}\left\|\hat{\mathbf{h}}_{\mathrm{MC}}\right\|^{2} s_{n}+\eta_{n, 1}+\eta_{n, 2}+\eta_{n, 3}+\eta_{n, 4} .
$$

Note that $\eta_{n, 1}$ and $\eta_{n, 3}$ are correlated, both to each other, and to the symbol $s_{n}$ and that $\eta_{n, 2}$ and $\eta_{n, 4}$ are uncorrelated to all other terms. To calculate the SNR in (13), one can split $\eta_{n, 1}$ and $\eta_{n, 3}$ into parts that correlate perfectly with $s_{n}$, and a part that is uncorrelated to $s_{n}$, as done in Section III-C.

\section{E. OSTBCs in Massive MIMO}

Because a massive MIMO BS has an abundance of transmit antennas, it generally has more options in the signal design compared to contemporary BSs. For example, the BS has, to a greater extent, the ability to dynamically change what STBC to use. If the BS is equipped with $M$ antennas, the size of the code (number of antenna ports) $n_{\mathrm{T}}$ can be changed to suite the scenario in question. If high reliability is needed, and there is little time/frequency diversity in the channel, the BS can choose a large $n_{\mathrm{T}}$ to compensate the lack of time/frequency diversity by adding spatial diversity. If the channel offers enough time/frequency diversity, a code with low diversity and high code rate may be used. The caveat here is, as we will see, that even if the BS may choose $n_{\mathrm{T}}$ to be any integer between 1 and $M$ in theory, a very large value of $n_{\mathrm{T}}$ is not possible or useful in practice.

There are limits to how high rate an OSTBC spanning $n_{\mathrm{T}}$ antenna ports can have. For example, no OSTBC can have a rate higher than 1 , and for $n_{\mathrm{T}}>1$, this rate is only achievable with $n_{\mathrm{T}}=2$ (the Alamouti code). The maximum rate of an OSTBC with $n_{\mathrm{T}}=2 m$ or $n_{\mathrm{T}}=2 m-1$, with $m$ being an integer is $\frac{m+1}{2 m}$. In particular, as $n_{\mathrm{T}}$ grows, the maximum rate approaches $1 / 2$ [21].

The second dimension of an OSTBC, the delay $\tau_{\mathrm{D}}$, becomes more important the larger $n_{\mathrm{T}}$ is, as $\tau_{\mathrm{D}} \leq \tau_{\mathrm{C}}-\tau_{\mathrm{P}}$ is required for the code to fit into one coherence interval. In general, for a fixed code rate $n_{\mathrm{S}} / \tau_{\mathrm{D}}$, delay increases quite fast with $n_{\mathrm{T}}$. The minimum delay grows especially fast when OSTBCs with optimal rates are considered. For example, the minimum delay of a maximum rate code with $n_{\mathrm{T}}=8$ antennas is $\tau_{\mathrm{D}}=56$ channel uses, and for a code with $n_{\mathrm{T}}=16$, the minimum delay is 11440 channel uses [25].

Hence, we have a practical limit to the code size $n_{\mathrm{T}}$. The limiting factor for massive MIMO, when it comes to choosing an OSTBC is the decoding delay together with the finite coherence interval, not the number of antenna ports. This means that increasing $n_{\mathrm{T}}$ stops being useful at some point, since the decoding delay is too long.

\section{IMPACT OF THE DIMENSION REDUCING MATRIX}

Let us now consider the transmission over the effective channel $\mathbf{h}$. The statistics of $\mathbf{h}$ depend on the choice of the DRM $\boldsymbol{\Phi}$ and the statistics of the physical channel $\mathbf{g}$ as indicated by (7). Apart from studying i.i.d. Rayleigh fading we also consider a correlated channel model which is described in Section IV-A. The choice of DRM and how the channel statistics affect this choice is discussed in Section IV-B.

\section{A. Channel Covariance Matrix}

To understand how correlation between antennas affects performance, we model the correlation of the antenna array with an exponential correlation matrix [26]. This model has the beauty of being parameterized by a single complex parameter, $r$, denoting the (complex) correlation between the channels of two neighboring antennas. The $(i, j)$ :th element of the covariance matrix $\mathbf{C}_{\mathbf{g}}$ is given by

$$
\mathbf{C}_{\mathbf{g}}(i, j)=\beta|r|^{|j-i|} e^{i \arg (r)(j-i)}
$$

with $|r| \leq 1$. This means that channels for antennas further apart have a smaller correlation, which is physically reasonable.

Two interesting special cases of this correlation matrix are when $r=0$ or $|r|=1$. For $r=0, \mathbf{C}_{\mathbf{g}}$ is a scaled identity matrix and hence corresponds to i.i.d. fading. If $|r|=1$, then all columns of $\mathbf{C}_{\mathbf{g}}$ are linearly dependent, so the correlation matrix has rank 1 . Note that for large arrays, even when $|r|$ is close to 1 , the correlation between antennas 
at moderate distance becomes negligible, as the correlation decays exponentially with the antenna distance.

The complex parameter $r=|r| e^{\mathrm{i} \arg (r)}$ depends on the magnitude $|r|$ and the $\operatorname{argument} \arg (r)$. In the numerical results, we fix $|r| \in[0,1]$ and let $\arg (r)$ vary depending on the user position. We set $\arg (r)$ to be the angle of incidence (as if a line-of-sight channel) from the user to the BS array. This means that we only need to specify $|r|$.

\section{B. Choosing the Dimension-Reducing Matrix}

Any choice of $\boldsymbol{\Phi}$ confines the effective channel $\mathbf{h}$ to the subspace spanned by the columns of $\boldsymbol{\Phi}$; the BS implicitly beamforms into this subspace. For physical channels $g$ in the approximate nullspace of $\boldsymbol{\Phi}$, the effective channel gains will be small. There is an intricate connection between the choice of DRM and the resulting SNR, since the DRM $\boldsymbol{\Phi}$ shows up at several places in (14). The question is how to choose a suitable DRM depending on, among other things, the chosen code and number of BS antennas. Note that there is no obvious "optimal" DRM here. One way of finding an upper bound on performance would be to assume perfect CSI at the BS; however, in this case, the BS would be able to beamform in a conventional manner (by for example multi-casting), making the comparison void.

When transmitting SI, the BS does not know who is listening; hence the choice of DRM should not depend on the physical channel g. However, if the BS has statistical knowledge of the channel, this could be used when constructing the DRM. Recall that we do not assume any channel knowledge, statistical or instantaneous, at the BS.

To illustrate the importance of the DRM, we compare three different strategies for choosing the DRM:

- The first DRM considered is the one derived in [8, Eq. (30)]. This matrix, denoted $\boldsymbol{\Phi}_{[8]}$, has several desirable properties: it ensures approximate omnidirectional transmission, equal output power on all antennas on the average and signals with low peak-to-average-power ratio.

- Second, we choose a random DRM:

$$
\boldsymbol{\Phi}_{\mathrm{RAND}} \triangleq\left[\begin{array}{ll}
\mathbf{I}_{n_{\mathrm{T}}} & \mathbf{0}_{n_{\mathrm{T}} \times\left(M-n_{\mathrm{T}}\right)}
\end{array}\right] \mathbf{Q},
$$

where $\mathbf{Q} \in \mathbb{C}^{M \times M}$ is an isotropically distributed unitary matrix [27], in order to make the matrix "as random as possible".

- Third, we choose the DRM as $n_{\mathrm{T}}$ evenly spaced columns in the $M$ dimensional discrete Fourier transform (DFT) matrix. That is, the columns with indices

$$
\frac{M}{2 n_{\mathrm{T}}}(2 n-1), n=1, \ldots, n_{\mathrm{T}} .
$$

We denote this matrix by $\boldsymbol{\Phi}_{\mathrm{DFT}}$.

The second and third choices are heuristic. The DRM $\boldsymbol{\Phi}_{\text {RAND }}$ demonstrates the performance of a matrix without any particular structure. This is a reasonable choice if the BS has no idea what effect the DRM has on the transmission. The motivation for $\boldsymbol{\Phi}_{\mathrm{DFT}}$ is that the columns of the DFT matrix corresponds to different angular directions. By spreading out the angles, at least one of them should work reasonably well for any given terminal. We expect $\boldsymbol{\Phi}_{[8]}$ to outperform the other two, as it is optimized. The main reason we present the other two DRMs is to show that a seemingly reasonable choice $\left(\boldsymbol{\Phi}_{\mathrm{DFT}}\right)$ can perform poorly, while a random matrix $\left(\boldsymbol{\Phi}_{\text {RAND }}\right)$ can perform well.

Remark: There are minor similarities between the DRM used here and the prebeamforming matrix used in [28]: both matrices can be built up from selected columns of the DFT matrix and simplify the channel estimation. However, the prebeamforming matrix in [28] has another purpose: to divide known users in the cell into groups based on the eigenspace of the users' covariance matrices. This is a completely different scenario than considered herein, where statistical CSI is available to the BS, the channel model is different, and payload data is transmitted.

Note that all three choices of DRMs are semi-unitary: $\boldsymbol{\Phi} \boldsymbol{\Phi}^{\mathrm{H}}=\mathbf{I}_{n_{\mathrm{T}}}$. For i.i.d. Rayleigh fading, $\mathbf{C}_{\mathbf{g}}=\beta \mathbf{I}_{M}$, this implies that the effective channel $\mathbf{h}$ will have the same statistics for any choice of DRM:

$$
\mathbf{C}_{\mathbf{h}}=\beta \boldsymbol{\Phi} \mathbf{I}_{M} \boldsymbol{\Phi}^{\mathrm{H}}=\beta \mathbf{I}_{n_{\mathrm{T}}} .
$$

Thus, all three choices are equivalent and the choice only makes a difference when $\mathbf{C}_{\mathbf{g}}$ is not a scaled identity matrix.

The cell edge SNR is defined as the SNR experienced by a terminal on the cell edge, if all power were transmitted from a single antenna in the array. Throughout the paper, we have a cell edge SNR of $-5 \mathrm{~dB}$.

To see the effects of the DRM, consider a correlation coefficient $|r|=0.9$ for two scenarios: one where the BS has $M=24$ antennas and uses code 2 , and one where the BS has $M=120$ antennas and uses code 8. Fig. 1 shows the cumulative distribution function (CDF) of the SNR (15) for uniformly distributed users on the cell edge when the BS is using different DRMs. To see the variation in performance of $\boldsymbol{\Phi}_{\text {RAND }}$, which is random by definition, Fig. 1 shows the best and the worst out of 10 realizations.

The difference in performance is solely due to the different DRM and how well these "match" the covariance matrix $\mathbf{C}_{\mathrm{g}}$. The randomness is due to user positions and the smallscale fading. We see that $\boldsymbol{\Phi}_{\text {DFT }}$ performs poorly here, giving some users very good performance, and some very poor. In general, SI should be available to as many users as possible, so preferably the curves should be vertical (and far to the right). That is, a spatially selective DRM, with a large (approximative) null space performs poorly when the terminals are uniformly distributed.

Interestingly, the random choice performs at a similar level as the optimized DRM in terms of symbol SNRs. That being said, $\boldsymbol{\Phi}_{\text {RAND }}$ does not satisfy, for example, the constraint necessary to ensure equal power through all antennas as $\boldsymbol{\Phi}_{[8]}$ does. In addition $\boldsymbol{\Phi}_{[8]}$ performs slightly better than $\boldsymbol{\Phi}_{\text {RAND }}$ for larger codes, as seen in Fig. 1b. Nevertheless picking a random DRM works relatively well. For larger codes, the performance of $\boldsymbol{\Phi}_{\mathrm{DFT}}$ improves, but is always considerably worse than both $\boldsymbol{\Phi}_{\text {RAND }}$ and $\boldsymbol{\Phi}_{[8]}$. This is due to the mismatch between $\boldsymbol{\Phi}_{\mathrm{DFT}}$ and the covariance matrix $\mathbf{C}_{\mathbf{g}}$. If the covariance matrix has a different structure or if users are distributed differently, the DFT choice might very well perform similar to or better than the other choices. 


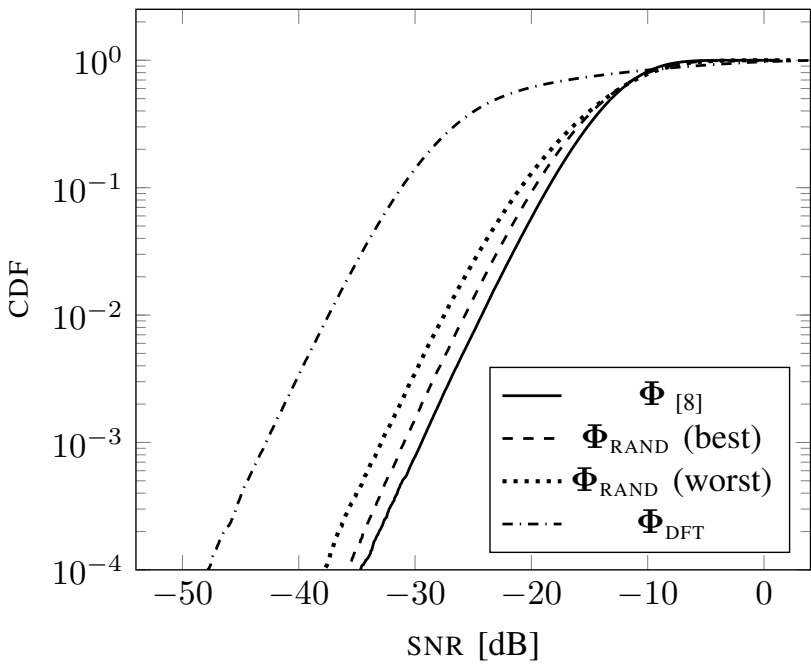

(a)

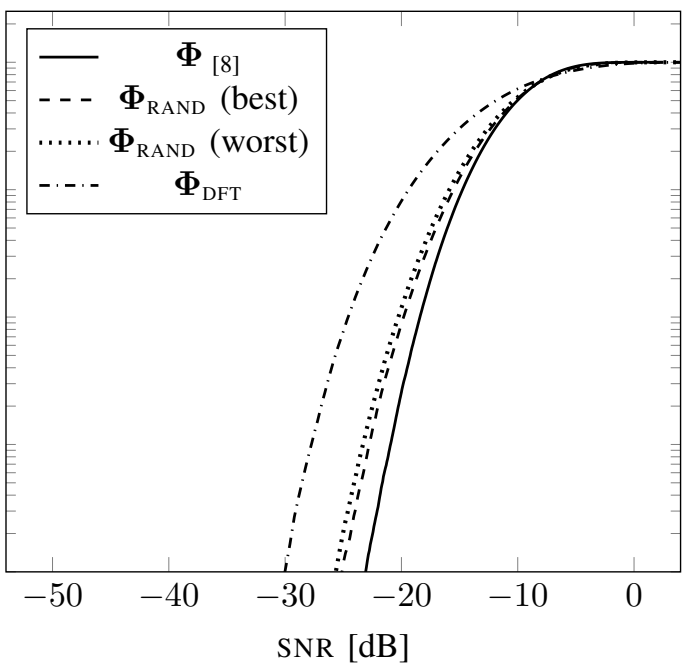

(b)

Fig. 1. Comparison of the three choices of DRM $\boldsymbol{\Phi}$ for two different codes in two different scenarios. We include two realizations of the random DRM, $\boldsymbol{\Phi}_{\mathrm{RAND}}$, to see how the performance differs between realizations. In this scenario we consider $|r|=0.9$ and a cell edge SNR of -5 dB. The terminals are uniformly distributed (in angle) on the cell edge. The DFT choice is poor, while the other choices perform more similar to each other. The randomness stems from the user positions as well as the small-scale fading. a) $M=24$ BS antennas, using code 2 ; b) $M=120$ BS antennas, using code 8 .

Looking at figures similar to Fig. 1 for different scenarios (different $M,|r|$, and codes, not included here) more general conclusions can be drawn. $\boldsymbol{\Phi}_{[8]}$ is a "one size fits all" DRM. It performs well for many choices of channel covariance matrices, codes and number of transmitting antennas. However, this does not mean that it is optimal in the sense of offering coverage to the largest number of terminals for any channel.

\section{Performance Metric}

To evaluate the performance of different codes in various settings, we consider outage rates instead of ergodic measures on capacity because of the limited number of diversity branches. It was shown in [29] that

$$
R^{*}(n, \epsilon)=C_{\epsilon}+\mathcal{O}\left(\frac{\log (n)}{n}\right),
$$

where $C_{\epsilon}$ denotes the outage capacity and $R^{*}(n, \epsilon)$ denotes the maximal achievable rate for block length $n$ and outage probability $\epsilon$. That is, the outage capacity is a good approximation to $R^{*}(n, \epsilon)$ if $n$ is large enough.

An additive white Gaussian noise (AWGN) channel with an SNR of $x$ can reliably support a maximum rate of $\log _{2}(1+x)$ [30, Section 5.4.1]. This means, conditioned on the channel estimate $\hat{\mathbf{h}}$ and assuming worst-case noise (Gaussian), the effective channel in (11) can support a maximum rate of

$$
\frac{n_{\mathrm{S}}}{\tau_{\mathrm{D}}} \log _{2}\left(1+\mathrm{SNR}^{\text {OSTBC }}\right) \text { bpcu. }
$$

Outage occurs if the used rate $R$ is larger than (18), i.e., if

$$
R>\frac{n_{\mathrm{S}}}{\tau_{\mathrm{D}}} \log _{2}\left(1+\mathrm{SNR}^{\text {OSTBC }}\right) .
$$

The received symbol SNR at the terminal depends on the realization of the channel estimate which in turn depends on the true channel. We assume independent channel realizations in each coherence interval and let $\mathrm{SNR}_{l}^{\mathrm{OSTBC}}$ denote the SNR experienced at the terminal in coherence interval $l$ when an OSTBC is used at the BS. Assuming coding over $L$ different coherence intervals, the average supported rate is

$$
\frac{1}{L} \sum_{l=1}^{L} \frac{n_{\mathrm{S}}}{\tau_{\mathrm{D}}} \log _{2}\left(1+\mathrm{SNR}_{l}^{\text {OSTBC }}\right) \text { bpcu. }
$$

The probability of outage when using a rate $R$ is then

$$
p_{\text {out }}^{\text {osTBC }}(R) \triangleq \operatorname{Pr}\left(R>\frac{1}{L} \sum_{l=1}^{L} \frac{n_{\mathrm{S}}}{\tau_{\mathrm{D}}} \log _{2}\left(1+\mathrm{SNR}_{l}^{\mathrm{OSTBC}}\right)\right) .
$$

For a given $\epsilon$, the outage capacity is defined as

$$
C_{\epsilon}^{\text {ostвC }} \triangleq \sup \left\{R: p_{\text {out }}^{\text {ostBC }}(R)<\epsilon\right\} .
$$

In order to take training into account, we define the outage rate as

$$
R_{\epsilon}^{\text {OSTBC }} \triangleq\left(\frac{\tau_{\mathrm{C}}-\tau_{\mathrm{P}}}{\tau_{\mathrm{C}}}\right) C_{\epsilon}^{\text {OSTBC }} \text { bpcu, }
$$

where we have scaled the outage capacity by the fraction of the coherence interval used for transmitting data.

Completely analogous to (19) we can define outage rates for general transmission and for transmission with a square OSTBC, using (17) and (16), respectively. We let $\operatorname{SNR}_{l}^{\text {general }}$ and $\mathrm{SNR}_{l}^{\text {square }}$ be the SNR experienced by the terminal in the $l:$ th coherence interval in the two cases. Performing identical calculations as above gives the corresponding outage rates

$$
R_{\epsilon}^{\text {general }} \triangleq\left(\frac{\tau_{\mathrm{C}}-\tau_{\mathrm{P}}}{\tau_{\mathrm{C}}}\right) C_{\epsilon}^{\text {general }} \text { bpcu, }
$$

and

$$
R_{\epsilon}^{\text {square }} \triangleq\left(\frac{\tau_{\mathrm{C}}-\tau_{\mathrm{P}}}{\tau_{\mathrm{C}}}\right) C_{\epsilon}^{\text {square }} \text { bpcu. }
$$

We expect that $R_{\epsilon}^{\text {general }} \geq R_{\epsilon}^{\text {OSTBC }}$, which we will quantify numerically in Section VI. 
One thing to note about the rate in (18) is that the reciprocal of the code rate $\frac{n_{\mathrm{S}}}{\tau_{\mathrm{D}}}$ is found in the numerator of SNR ${ }^{\mathrm{OSTBC}}$. For low SNR this means that the rate in (18) is almost independent of the code rate, since $\log (1+x) \approx x$ if $x \ll 1$.

\section{Simulations}

We consider the OSTBCs listed in Table I and compare the outage rates of these, as defined in (19), in different scenarios. We will see how the performance varies depending on the number of BS antennas, $M$, and the correlation coefficient $|r|$. In the end, we will also compare the results of the single-cell case to that of a multi-cell case.

Throughout the simulations, the outage probability $\epsilon=0.01$ is fixed. The terminals are distributed uniformly in a disk with radius 1 in the single-cell case and in a regular hexagon with circumradius 1 in the multi-cell case. Both in the single and multi-cell case, a small disk with radius 0.035 around the BS is excluded. Large-scale fading consists of distance-dependent path loss with exponent 3.8 and the cell edge SNR is set to $-5 \mathrm{~dB}$. The coherence interval consists of $\tau_{\mathrm{C}}=256$ symbols. $^{2}$ We only consider DRM $\boldsymbol{\Phi}_{[8]}$, as it performs well in all tested scenarios.

Initially, we will only consider transmission over one coherence interval; hence no time/frequency diversity is available. In Sections VI-C, VI-D and VI-E, the BS is allowed to code over several coherence intervals. Results from the multi-cell scenario is presented in Section VI-F.

\section{A. Pilot Energy Optimization}

To facilitate fair comparisons, all transmission strategiesno matter what code or DRM-will have the same energy budget (the amount of energy spend in one coherence interval). We consider a heuristic way of optimizing the pilot energy, $\tau_{\mathrm{P}} \rho_{\mathrm{P}}$, by maximizing the outage rate of a simplified scenario, with the same parameters. We only optimize over $\rho_{\mathrm{P}}$ since [31, Theorem 1] ensures that the outage rate is maximized when $\tau_{\mathrm{P}}=n_{\mathrm{T}}$

To perform the heuristic optimization, the BS assumes that a square OSTBC is used, the channel coefficients are i.i.d., and that the SNR at the user only depends on the large-scale fading, which has a known distribution. Note that the optimization can be done regardless of the validity of these assumptions. Now, with these assumptions, the outage rate is given by (21). For an outage probability of $\epsilon$, the BS considers the large-scale coefficient associated with the $\epsilon$ percentile, denoted $\beta_{\epsilon}$. That is, a fraction $1-\epsilon$ of the large-scale fading coefficients is larger than $\beta_{\epsilon}$ and a fraction $\epsilon$ is smaller than $\beta_{\epsilon}$. The BS then considers the outage rate in (21) and calculates the value of $\rho_{\mathrm{P}}$ such that this outage rate is maximized. For our purpose, this heuristic method does not necessarily result in the optimal pilot energy because the resulting symbol SNR (15) when using the codes in Table I will not equal the symbol SNR in (16). This method, however, does not require any CSI at the BS.

\footnotetext{
${ }^{2}$ The specific number was chosen to be a power of two, to simplify some of the simulations. It is still in the same order of magnitude as the smallest scheduling unit in LTE (168) and the coherence interval for a coherence time of $1 \mathrm{~ms}$ and a coherence bandwidth of a few hundred $\mathrm{kHz}$.
}

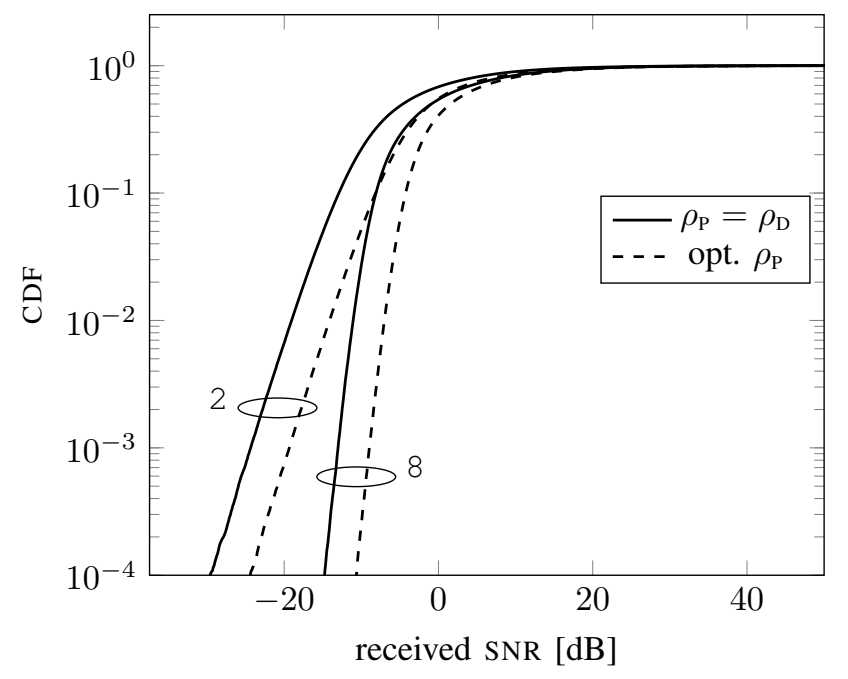

Fig. 2. The received SNR (15) with and without optimizing the pilot energy for code 2 and code 8 . We see that heuristically optimizing the pilot energy is beneficial.

Now, let us see the effect of the optimization, by comparing the performance to the baseline: spending the minimum amount of symbols on pilots $\left(\tau_{\mathrm{P}}=n_{\mathrm{T}}\right)$, while keeping the transmission power constant over the entire coherence interval $\left(\rho_{\mathrm{D}}=\rho_{\mathrm{P}}\right)$. We consider the case of uncorrelated channels $(r=0)$ here, but the same conclusions can be drawn when looking at correlated channels. Fig. 2 shows the CDF for the SNRs with and without optimizing the pilot energy for two different codes. As seen, the baseline lags behind considerably for both codes, and the optimization proves useful.

In light of these results, all presented outage rates in the remainder of the paper have been optimized as presented in this section, which means that all codes use the minimum number of pilot symbols $\left(\tau_{\mathrm{P}}=n_{\mathrm{T}}\right)$. As a consequence, the pilot symbols will be transmitted with considerably more power than the subsequent data symbols.

\section{B. Without Time/Frequency Diversity}

First, let us consider the case of i.i.d. Rayleigh fading. The outage rates for the considered codes are shown in Fig. 3a. As indicated by theoretical results, the performance does not depend on the number of antennas (nor the chosen DRM, as long as it is semi-unitary). In the same graph, shown with filled markers, are the achievable outage rates for (20). We first note that the two bounds are tight, not only for codes 1 and 2 as we mentioned in Section III-C, but also for rectangular codes with code rate less than one, as seen by the overlapping markers. This is because the SNR is low here, so the decrease in code rate is compensated by the increase in SNR.

When time/frequency diversity is scarce, spatial diversity is extremely useful. Studying Fig. 3a more closely reveals that adding just a little spatial diversity can have a big impact, and the effect is more prominent the smaller outage probability, $\epsilon$, we require. Increasing the diversity order, going from 1 to 2 (effectively doubling the number diversity branches) gives a fivefold increase in outage rate. As we again double the diversity order, from 2 to 4 , the rate is doubled. Doubling yet 


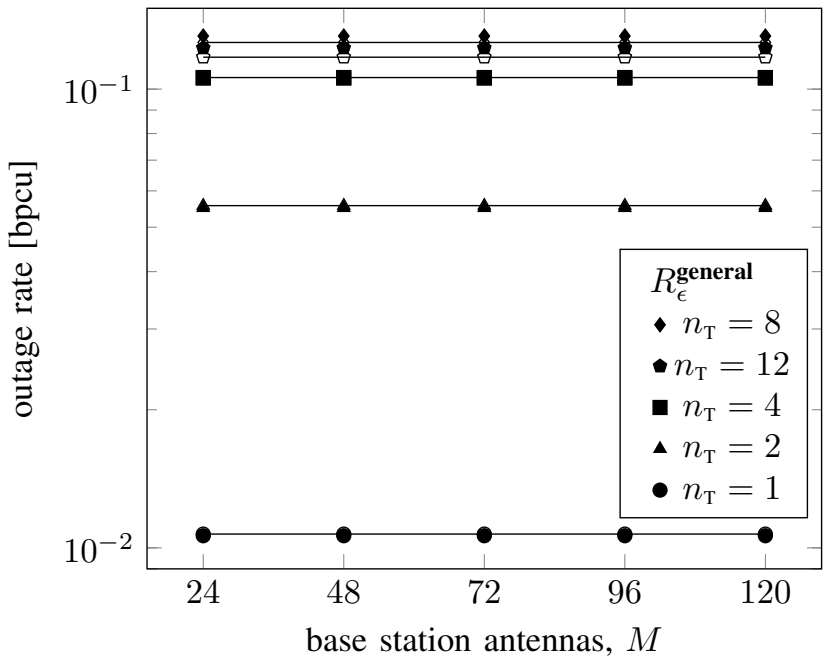

(a)

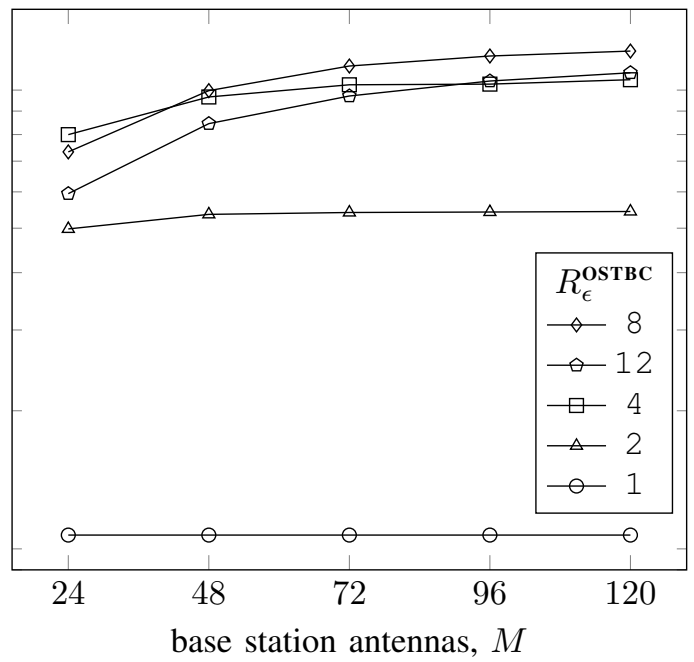

(b)

Fig. 3. The outage rates for uncorrelated and correlated fading. In both scenarios, the spatial diversity pays off a great deal, but reaches a clear point of diminishing return for the larger codes. a) The two outage rates (19) and (20) are very tight for most choices of $n_{\mathrm{T}}$, but we see a slightly larger difference for $n_{\mathrm{T}}=12$, as the markers do not overlap completely. b) A correlated channel with correlation coefficient $|r|=0.9$ is considered. As the number of antennas grows, the correlation becomes negligible since $|r|^{M}$ decays quickly and the outage rate approaches that of the uncorrelated channel. The correlation strikes the larger codes harder when the number of BS antennas is small.

again, up to diversity order 8 , gives a moderate increase of about 10 percent. The diminishing return of diversity is most apparent when comparing the two larger codes. In Fig. 3a, the largest code does not give the highest rate. The reasons for this are twofold: First, the benefit of the extra spatial diversity is not big enough to counteract the effect of the increased pilot overhead. Second, the heuristic optimization works better for smaller codes (as the approximation of being square is more accurate). Around the point of $n_{\mathrm{T}}=10$, the effect of increasing the spatial diversity is overcome by the increase in pilot overhead, and thus larger codes are not useful. This is a consequence of the relatively short coherence interval, and the choice of outage probability $\epsilon$. Larger codes could still be useful in a scenario with longer coherence intervals or lower outage probability.

Fig. $3 b$ shows the outage rates for correlated channels with correlation coefficient $|r|=0.9$. When the channels are correlated, the outage rate decreases, as can be seen by comparing Fig. 3a and Fig. 3b. This drop in performance is due to the DRM not matching the channel covariance matrix when the channels are correlated, while any semi-unitary matrix matches the channel covariance matrix when the channels are uncorrelated. When the number of BS antennas grows, the outage rate tends to that of the uncorrelated channel. This is because as the array grows, more antennas are further away from each other which decreases the correlation between the channels. Since $|r|^{M}$ decays quickly, only a moderate number of antennas is needed to mitigate even quite large correlation coefficients. The smaller codes struggle because of the lack of diversity, while the larger code gets punished by the symbols spent on pilots, as well as the optimization.

\section{With Time/Frequency Diversity}

Choosing the code giving the maximum rate, we see from Fig. 3a that the BS can convey about $0.12 \mathrm{bpcu}$ for the chosen scenario. Over one coherence interval this means about 30 bits. If the BS needs to convey more bits with the same outage probability, more resources have to be allocated.

As we have seen previously, when the channel offers no time/frequency diversity, the larger codes tend to give a higher rate, since the spatial diversity from the code is so valuable. When the channel offers more time/frequency diversity, however, the spatial diversity from the code decreases in value. This is observed in Fig. 4, where the outage rate for each code is shown as a function the number of coherence intervals, $L$, the BS codes over. Each coherence interval sees an independent channel realization, and hence the time/frequency diversity order is $L$.

In general, larger codes saturate faster, as they reach the point of diminishing returns quicker. They also saturate at a lower rate, because of the lower code rate, $n_{\mathrm{S}} / \tau_{\mathrm{D}}$. Code 1 gains a lot from the extra time/frequency diversity and quickly catches up to the other codes as the number of diversity branches increases. As $L$ tends to infinity, in which case ergodic capacity would be a relevant metric, performance is determined by the code rate, and hence, the smaller codes with higher code rates are superior. Note that the Alamouti code is better than 1 for all values considered, as it offers more diversity and the same code rate.

\section{Fixed Message Length}

Ultimately, what code to choose depends on how much information the BS needs to convey to the terminals. Consider a message of $N_{\mathrm{b}}$ bits. The BS aims to reach 99 percent (cf. $\epsilon=0.01$ ) of the terminals with this message. How many coherence intervals must be allocated to make this happen? 


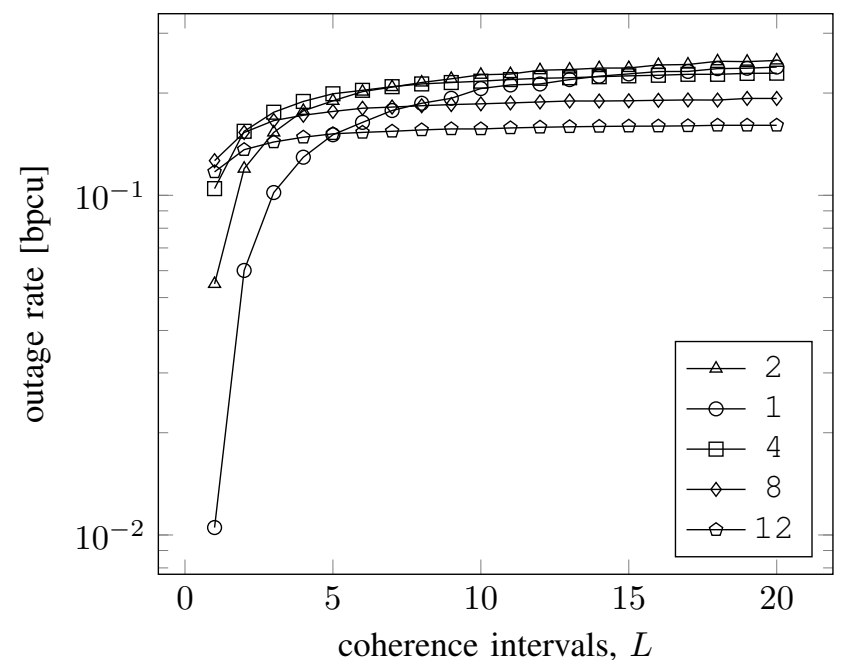

Fig. 4. The outage rate (19) when coding over several coherence intervals. The smaller codes performs poorly when only a few coherence intervals are allocated for transmission, because of the lack of diversity. As the number of allocated coherence intervals increases, the spatial diversity of the code matters less, and the codes with the highest code rate perform the best.

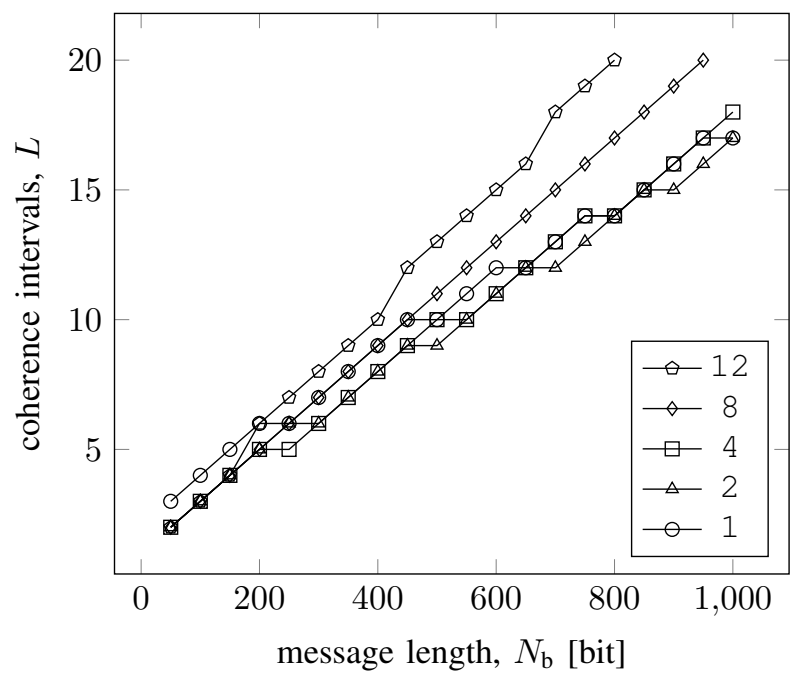

Fig. 5. The minimum number of coherence intervals needed to convey message of $N_{\mathrm{b}}$ bits with outage probability less than $\epsilon=0.01$. For short messages, the larger codes provide sufficient rate, but as the message gets longer, the smaller code rate is too costly. For large messages, the base station needs to allocate more coherence intervals, providing time/frequency diversity, making the spatial diversity less useful.

We use the outage rates in Fig. 4 and see how many bits can be conveyed using the different codes. Depending on the size of the message, $N_{\mathrm{b}}$, the BS has to allocate different number of coherence intervals for each code. The minimum number of coherence intervals required for each code is shown in Fig. 5.

For many choices of message length $N_{\mathrm{b}}$, several codes might need the same number of coherence interval to convey the message, as seen by the overlapping curves in Fig. 5. In this case, we would choose the largest code, since the added diversity will make the received SNR more reliable (slightly lower outage probability). The general trend is that larger codes are preferred for short messages, when few coherence intervals are needed, and smaller codes are preferred for long

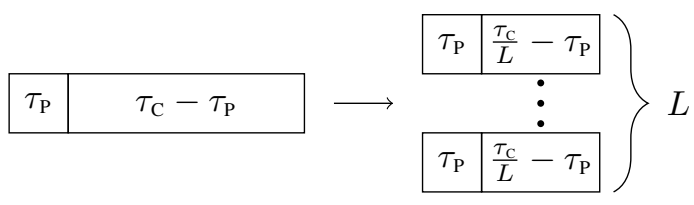

Fig. 6. The $\tau_{\mathrm{C}}$ channel uses does not necessarily have to be allocated in the same coherence interval. By spreading the SI over $L$ coherence intervals we get time/frequency diversity at a cost of increased pilot overhead.

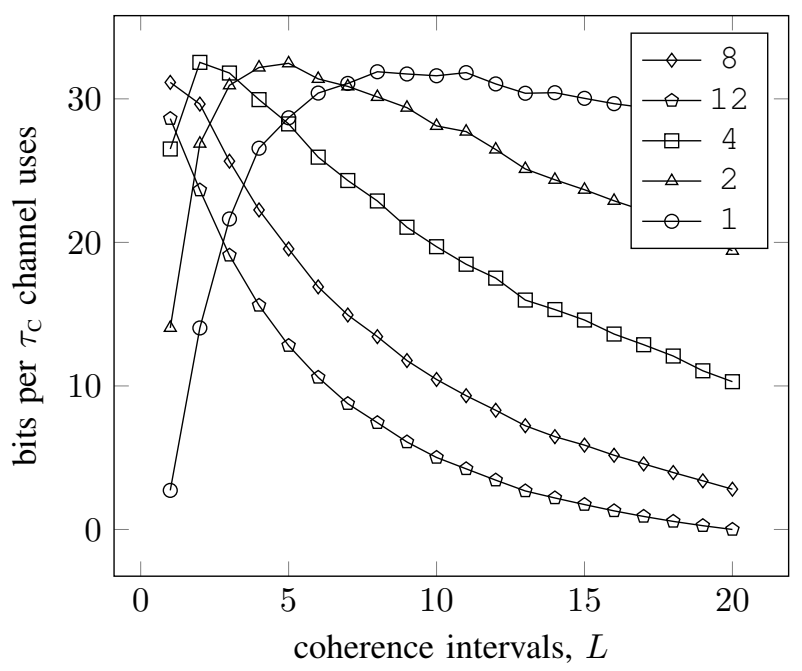

Fig. 7. The total number of bits transferred over $\tau_{\mathrm{C}}$ channel uses for the different codes in Table I when transmission is split over several coherence intervals. Larger codes are punished quickly because of the relatively large pilot overhead, while smaller codes see an improvement due to their lack of diversity. However, approximately the same maximum is achieved regardless of what code is used.

messages, as the many allocated coherence intervals provide enough diversity for the outage probability to be small. To take specific examples from Fig. 5, we see that code 4 is preferred when $N_{\mathrm{b}}=250$ and code 2 is preferred when $N_{\mathrm{b}}=500$.

\section{E. Fixed Number of Channel Uses}

We now allow for a coherence interval to carry both SI and other data. That is, the entire coherence interval does not necessarily have to be dedicated for SI. Although one coherence interval may carry both SI and other data, we do not multiplex spatially within one channel use as in [13]. We analyze whether splitting up SI over several coherence intervals can improve performance.

Consider having a total of $\tau_{\mathrm{C}}=256$ channel uses dedicated to transmitting SI. If these channel uses are spread over several coherence intervals, we can code over several channel realizations, and hence the time/frequency diversity increases. On the other hand, we have to transmit downlink pilots in each of the coherence intervals, so fewer channel uses can actually be used for data. To be more precise: spreading the SI over $L$ coherence intervals will leave $\tau_{\mathrm{C}}-L \tau_{\mathrm{P}}$ channel uses for data, depicted in Fig. 6. This then yields a trade-off, once again, between diversity and pilot overhead, also mentioned in [32]. We stress that the minimum number of pilot symbols is used, i.e., $\tau_{\mathrm{P}}=n_{\mathrm{T}}$. 
Fig. 7 shows the total number of bits each code can transfer over 256 channel uses, when transmission is spread over $L$ coherence intervals. The first thing to note is that all codes can, approximately, transfer the same amount of information, 31 bits, over $\tau_{\mathrm{C}}$ channel uses. This tells us that all codes perform similarly if the BS is allowed to spread the SI over several coherence intervals. Second, the maximum for all codes occurs when the total number of diversity branches $L n_{\mathrm{T}}$ is between 8 and 10. This means that, for this particular scenario, there is a tipping point at around $L n_{\mathrm{T}}=9$ diversity branches: more branches require too much pilot overhead, fewer branches give too little diversity. This is why code 12 performs worse than the others: the diversity is already saturated. The same phenomenon is observed for other scenarios as well, although the location of the tipping point differs. For a longer coherence interval or for a lower outage probability, the optimal number of diversity branches increases. As a consequence, the tipping point will move to the right.

\section{F. Multi-cell Setup}

We now consider a multi-cell setup with 19 cells: 18 interfering cells, and the home cell, in the center. We consider three different pilot-reuse factors and compare the outage rate when using different OSTBCs. Apart from now considering multiple cells, the setup is identical to that in Fig. 3, with the same correlation factor of 0.9 and with $M=120$ BS antennas. There are three important differences compared to the singlecell case, as mentioned in Section III-D: i) Contaminating cells that use the same pilots interfere with the channel estimation. This can be mitigated by increasing the pilot reuse. ii) The data transmitted by other cells increase interference in the symbol detection, and is independent of the pilot reuse. iii) An increased pilot reuse requires longer pilots and therefore increases the pilot overhead.

In Fig. 8, we see that the pilot reuse has a huge effect on the outage rates in a multi-cell system. When all cells use the same pilots, the outage rate is only a small fraction of what it is for the single-cell case. For pilot reuse 3 or 4 , the outage rate is more similar to that of the single-cell. To make comparison fair here the shape of the single cell is hexagonal.

A secondary effect that also lowers the outage rates for the multi-cell setup is that the heuristic optimization in Section VI-A does not work as well as in the single-cell setup. This is because the effective SNR experienced near the cell edge is much lower than what the heuristic method assumes (since it ignores all inter-cell interference). As a consequence, it is actually better to not optimize when using reuse 1 in our case.

\section{CONCLUSION}

Downlink transmission in massive MIMO without CSI at the base station is necessary for conveying system information to the terminals in the cell. A massive MIMO base station can outperform a single-antenna base station, with the same power constraint, for correlated and uncorrelated channels. Hence, conveying system information without CSI is not a show-stopper for massive MIMO. As the number of diversity

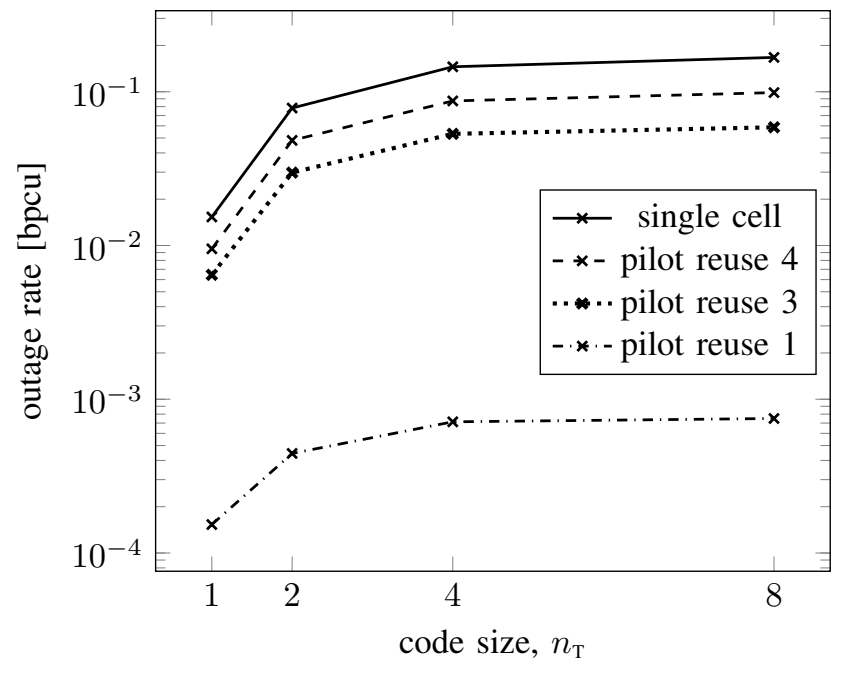

Fig. 8. Outage rates in a multi-cell systems with different pilot reuse for the four smallest codes in Table I. The interference from cells using the same pilots is very strong in the case of pilot reuse 1 , leading to a very low rate.

branches of the channel increases the benefit of the spatial diversity provided by the code decreases, making the larger codes primarily useful when time/frequency diversity is low. To convey short messages of a few hundred bits, less timefrequency resources are required and increased reliability can be provided if the base station uses codes which provide spatial diversity.

\section{REFERENCES}

[1] T. L. Marzetta, E. G. Larsson, H. Yang, and H. Q. Ngo, Fundamentals of Massive MIMO. Cambridge: Cambridge University Press, 2016.

[2] X. Gao, O. Edfors, F. Rusek, and F. Tufvesson, "Massive MIMO performance evaluation based on measured propagation data," IEEE Transactions on Wireless Communications, vol. 14, no. 7, pp. 3899 3911, Jul. 2015

[3] P. Harris, S. Zang, A. Nix, M. Beach, S. Armour, and A. Doufexi, "A distributed massive MIMO testbed to assess real-world performance and feasibility," in 2015 IEEE 81st Vehicular Technology Conference (VTC Spring), May 2015, pp. 1-2.

[4] C. Shepard, H. Yu, N. Anand, E. Li, T. Marzetta, R. Yang, and L. Zhong, "Argos: Practical many-antenna base stations," in Proceedings of the 18th Annual International Conference on Mobile Computing and Networking, ser. Mobicom '12. New York, NY, USA: ACM, 2012, pp. 53-64.

[5] J. Vieira, S. Malkowsky, K. Nieman, Z. Miers, N. Kundargi, L. Liu, I. Wong, V. Öwall, O. Edfors, and F. Tufvesson, "A flexible 100-antenna testbed for massive MIMO," in 2014 IEEE Globecom Workshops (GC Wkshps), Dec. 2014, pp. 287-293.

[6] E. Björnson, E. G. Larsson, and T. L. Marzetta, "Massive MIMO Ten myths and one critical question," IEEE Communications Magazine, vol. 54, no. 2, pp. 114-123, 2016.

[7] C. Shepard, A. Javed, and L. Zhong, "Control channel design for manyantenna MU-MIMO," in Proceedings of the 21st Annual International Conference on Mobile Computing and Networking, ser. MobiCom '15. New York, NY, USA: ACM, 2015, pp. 578-591.

[8] X. Meng, X. Gao, and X. G. Xia, "Omnidirectional precoding based transmission in massive MIMO systems," IEEE Transactions on Communications, vol. 64, no. 1, pp. 174-186, Jan. 2016.

[9] X. Meng, X.-G. Xia, and X. Gao, "Omnidirectional space-time block coding for common information broadcasting in massive MIMO systems," CoRR, vol. abs/1610.07771, Oct. 2016.

[10] X. G. Xia and X. Gao, "A space-time code design for omnidirectional transmission in massive MIMO systems," IEEE Wireless Communications Letters, vol. PP, no. 99, pp. 1-1, 2016 
[11] D. Qiao, H. Qian, and G. Y. Li, "Broadbeam for massive MIMO systems," IEEE Transactions on Signal Processing, vol. 64, no. 9, pp 2365-2374, May 2016

[12] Ericsson, "On forming wide beams," Ericsson, Spokane, WA, USA, Tech. Rep. R1-1700772, Jan. 2017.

[13] E. G. Larsson and H. V. Poor, "Joint beamforming and broadcasting in massive MIMO," IEEE Transactions on Wireless Communications, vol. 15, no. 4, pp. 3058-3070, Apr. 2016.

[14] Z. Xiang, M. Tao, and X. Wang, "Massive MIMO Multicasting in Noncooperative Cellular Networks," IEEE Journal on Selected Areas in Communications, vol. 32, no. 6, pp. 1180-1193, Jun. 2014.

[15] A. F. Molisch, V. V. Ratnam, S. Han, Z. Li, S. L. H. Nguyen, L. Li, and K. Haneda, "Hybrid Beamforming for Massive MIMO - A Survey," arXiv:1609.05078 [cs, math], Sep. 2016

[16] M. Karlsson and E. G. Larsson, "On the operation of massive MIMO with and without transmitter CSI," in 2014 IEEE 15th International Workshop on Signal Processing Advances in Wireless Communications (SPAWC), Jun. 2014, pp. 1-5.

[17] M. Karlsson, E. Björnson, and E. G. Larsson, "Broadcasting in massive MIMO using OSTBC with reduced dimension," in 2015 International Symposium on Wireless Communication Systems (ISWCS), Aug. 2015 pp. 386-390

[18] E. G. Larsson and P. Stoica, Space-Time Block Coding for Wireless Communications. Cambridge: Cambridge University Press, 2003.

[19] V. Tarokh, H. Jafarkhani, and A. R. Calderbank, "Space-time block codes from orthogonal designs," IEEE Transactions on Information Theory, vol. 45, no. 5, pp. 1456-1467, Jul. 1999.

[20] S. M. Alamouti, "A simple transmit diversity technique for wireless communications," IEEE Journal on Selected Areas in Communications, vol. 16, no. 8, pp. 1451-1458, Oct. 1998.

[21] X.-B. Liang, "Orthogonal designs with maximal rates," IEEE Transac tions on Information Theory, vol. 49, no. 10, pp. 2468-2503, Oct. 2003.

[22] S. Das and B. S. Rajan, "Low-delay, high-rate nonsquare complex orthogonal designs," Information Theory, IEEE Transactions on, vol. 58, no. 5, pp. 2633-2647, 2012.

[23] S. M. Kay, Fundamentals of Statistical Signal Processing, Volume I: Estimation Theory, 1st ed. Englewood Cliffs, N.J: Prentice Hall, Apr 1993.

[24] M. Medard, "The effect upon channel capacity in wireless communications of perfect and imperfect knowledge of the channel," IEEE Transactions on Information Theory, vol. 46, no. 3, pp. 933-946, May 2000.

[25] S. S. Adams, N. Karst, and J. Pollack, "The minimum decoding delay of maximum rate complex orthogonal space time block codes," IEEE Transactions on Information Theory, vol. 53, no. 8, pp. 2677-2684, Aug. 2007.

[26] S. L. Loyka, "Channel capacity of MIMO architecture using the exponential correlation matrix," IEEE Communications Letters, vol. 5, no. 9 , pp. 369-371, Sep. 2001

[27] T. L. Marzetta and B. M. Hochwald, "Capacity of a mobile multipleantenna communication link in Rayleigh flat fading," IEEE Transaction on Information Theory, vol. 45, no. 1, pp. 139-157, Jan. 1999.

[28] A. Adhikary, J. Nam, J. Y. Ahn, and G. Caire, "Joint spatial division and multiplexing: The large-scale array regime," IEEE Transactions on Information Theory, vol. 59, no. 10, pp. 6441-6463, Oct. 2013.

[29] W. Yang, G. Durisi, T. Koch, and Y. Polyanskiy, "Quasi-static multipleantenna fading channels at finite blocklength," IEEE Transactions on Information Theory, vol. 60, no. 7, pp. 4232-4265, Jul. 2014.

[30] D. Tse and P. Viswanath, Fundamentals of Wireless Communication. Cambridge: Cambridge University Press, 2005.

[31] H. V. Cheng, E. Björnson, and E. Larsson, "Optimal Pilot and Payload Power Control in Single-Cell Massive MIMO Systems," IEEE Transactions on Signal Processing, vol. PP, no. 99, pp. 1-1, 2016.

[32] W. Yang, G. Durisi, T. Koch, and Y. Polyanskiy, "Diversity versu channel knowledge at finite block-length," in 2012 IEEE Information Theory Workshop, Sep. 2012, pp. 572-576.

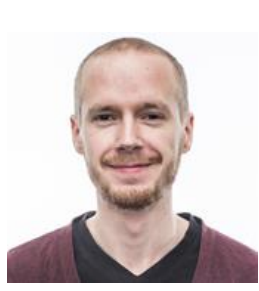

Marcus Karlsson received the M.Sc in electrical engineering in 2013 from Linköping university, where he is pursuing a Ph.D degree with the Division of Communication Systems at the Department of Electrical Engineering. His main research interest are different aspects of Massive MIMO, such as physical layer security, with a focus on jamming, and initial access, with a focus on transmission without channel knowledge at the base station.

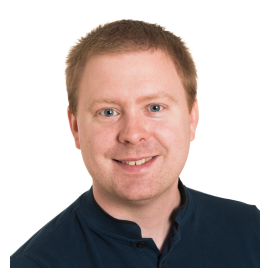

Emil Björnson (S'07, M12, SM17) received the M.S. degree in Engineering Mathematics from Lund University, Sweden, in 2007. He received the Ph.D. degree in Telecommunications from KTH Royal Institute of Technology, Sweden, in 2011. From 2012 to mid 2014, he was a joint postdoc at the AlcatelLucent Chair on Flexible Radio, SUPELEC, France, and at KTH. He joined Linköping University, Sweden, in 2014 and is currently Senior Lecturer and Docent at the Division of Communication Systems.

He performs research on multi-antenna communications, Massive MIMO, radio resource allocation, energy-efficient communications, and network design. He is on the editorial board of the IEEE TRANSACTIONS ON COMMUNICATIONS and the IEEE TRANSACTIONS ON GREen COMmunications AND Networking. He is the first author of the textbooks Massive MIMO Networks: Spectral, Energy, and Hardware Efficiency (2017) and Optimal Resource Allocation in Coordinated Multi-Cell Systems (2013). He is dedicated to reproducible research and has made a large amount of simulation code publicly available.

Dr. Björnson has performed MIMO research for more than ten years and has filed more than ten related patent applications. He received the 2016 Best $\mathrm{PhD}$ Award from EURASIP, the 2015 Ingvar Carlsson Award, and the 2014 Outstanding Young Researcher Award from IEEE ComSoc EMEA. He has co-authored papers that received best paper awards at WCSP 2017, IEEE ICC 2015, IEEE WCNC 2014, IEEE SAM 2014, IEEE CAMSAP 2011, and WCSP 2009.

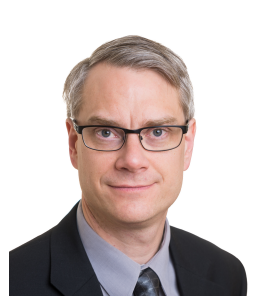

Erik G. Larsson (S99M03SM10F16) received the Ph.D. degree from Uppsala University, Uppsala, Sweden, in 2002

$\mathrm{He}$ is currently Professor of Communication Systems at Linköping University (LiU) in Linköping, Sweden. He was with the Royal Institute of Technology $(\mathrm{KTH})$ in Stockholm, Sweden, the University of Florida, USA, the George Washington University, USA, and Ericsson Research, Sweden. In 2015 he was a Visiting Fellow at Princeton University, USA for four months. His main professional interests are within the areas of wireless communications and signal processing. He has co-authored some 130 journal papers on these topics, and he is co-author of the two Cambridge University Press textbooks Space-Time Block Coding for Wireless Communications (2003) and Fundamentals of Massive MIMO (2016). He is co-inventor on 16 issued and many pending patents on wireless technology.

He was Associate Editor for, among others, the IEEE Transactions on Communications (2010-2014) and the IEEE Transactions on Signal Process ing (2006-2010). From 2015 to 2016 he served as chair of the IEEE Signa Processing Society SPCOM technical committee, and in 2017 he is the past chair of this committee. From 2014 to 2015 he served as chair of the steering committee for the IEEE Wireless Communications Letters. He was the Genera Chair of the Asilomar Conference on Signals, Systems and Computers in 2015, and its Technical Chair in 2012. He is a member of the IEEE Signal Processing Society Awards Board during 2017-2019.

$\mathrm{He}$ received the IEEE Signal Processing Magazine Best Column Award twice, in 2012 and 2014, the IEEE ComSoc Stephen O. Rice Prize in Communications Theory in 2015, and the IEEE ComSoc Leonard G. Abraham Prize in 2017. He is a Fellow of the IEEE. 